\title{
Parasites in stray dogs from Italy: prevalence, risk factors and management concerns
}

\author{
Claudio De Liberato ${ }^{1}$, Federica Berrilli², Livio Odorizi ${ }^{3}$, Roberto Scarcella ${ }^{4}$, Marco Barni ${ }^{5}$, \\ Cristina Amoruso ${ }^{1}$, Alessia Scarito ${ }^{1}$, Margherita Montalbano Di Filippo², Andrea Carvelli1* \\ Francesca lacoponi ${ }^{1}$ and Paola Scaramozzino ${ }^{1}$
}

\author{
${ }^{1}$ Istituto Zooprofilattico Sperimentale del Lazio e della Toscana "M. Aleandri", 00178 Roma, Italy; ${ }^{2}$ Dipartimento di Scienze Cliniche \\ e Medicina traslazionale, 00133 Roma, Italy; ${ }^{3} \mathrm{La}$ Fattoria di Tobia Shelter, 00036 Palestrina (Roma), Italy; ${ }^{4}$ Private Practitioner Vet, 00100 \\ Roma, Italy; ${ }^{5}$ ASL Roma 5, 00036 Palestrina (Roma), Italy
}

\begin{abstract}
Parasites are among the most common pathogens recorded in dogs. High prevalence rates are usually reported in stray and shelter dogs, as these animals are less likely to be tested and treated. In this study a survey was carried out on intestinal and lung parasites of stray dogs at the moment of the admission in a shelter in Central Italy. In the period June 2014-June 2015, 262 individual faecal samples were examined. Twelve parasitic taxa were detected. Helminths were more prevalent than protozoa (61.8\% and 25.6\%, respectively). Ancylostomatidae showed the higher prevalence (40.5\%), followed by Giardia duodenalis (21.4\%), Toxocara canis (20.6\%) and Trichuris vulpis (17.6\%). Angiostrongylus vasorum was the most prevalent lungworm (12.6\%), Crenosoma vulpis being recorded just in one dog. G. duodenalis had a high prevalence, probably because of the diagnostic techniques adopted, actually the most sensitive available for its detection. Nevertheless, the zoonotic potential of this parasite in dogs appears reduced, as all the isolates were identified as C (24.0\%) and D (76.0\%) dog specific assemblages. Taeniidae constituted a high potential zoonotic risk, as from the eggs it is impossible to exclude they were Echinococcus granulosus, the most relevant zoonotic parasite in Europe. The present study underline the importance of stray dogs control, aimed at preventing or minimizing parasitic spread and zoonotic transmission.
\end{abstract}

\section{Keywords}

Dog, parasites, zoonosis, risk factors, Italy

\section{Introduction}

Intestinal and lung parasites are among the most common pathogens recorded in dogs worldwide (Little et al. 2009; Mirzaei and Fooladi 2012; Al-Sabi et al. 2013; Riggio et al. 2013). Their health impact is significant both for the clinical disorders in dogs (Claerebout et al. 2009; Riggio et al. 2013) and for the zoonotic potential of some of them (Sager et al. 2006; Claerebout et al. 2009; Mirzaei and Fooladi 2012; Dopchiz et al. 2013; Mateus et al. 2014). Parasites are a common occurrence in all dog categories (i.e. household, farm, stray, shelter, hunting, etc.), although with different prevalence (Palmer et al. 2008; Claerebout et al. 2009; Little et al. 2009; Martins et al. 2012; Mirzaei and Fooladi 2012; Al-Sabi et al. 2013; Dopchiz et al. 2013; Traversa et al. 2017), higher ones being generally reported in stray and shelter dogs, less likely to be tested and treated, often exposed to high environmental faecalization and sometimes immunosuppressed because of malnutrition or stress (Palmer et al. 2008; Claerebout et al. 2009).

Intestinal nematodes and protozoa are usually the most prevalent parasites, given their direct life cycle and the ability of eggs/cysts to remain infective for long periods once shed in soil, while parasites with complex life cycles are generally recorded with lower prevalence (Palmer et al. 2008; Martins et al. 2012; Zanzani et al. 2014; Traversa et al. 2017).

Few data regarding parasites in stray dogs are available in literature, in particular concerning the European Union countries. Since stray dogs are not subject to any antiparasitic treatment, can move over long distances and usually live in synanthropic conditions, their environmental faecalization can represent a source of parasitic infection for people, owned dogs and other 
animal species (Martins et al. 2012; Mateus et al. 2014). Moreover, parasites in stray dogs represent a relevant sanitary and management concern once dogs are captured and transferred to refuge shelters, where high animal density facilitates transmission and spread of parasites with direct life cycles.

In Italy, according to national rules, the sanitary control of captured stray dogs is mandatory, concerning rabies and some zoonotic parasitic infections (e.g. Echinococcus granulosus and Leishmania infantum). In 2015, in Italy more than 100,000 roaming stray dogs were captured on a total of 600,000 estimated population (Italian Ministry of Health, unpublished data).

The aim of this paper is to evaluate the prevalence of intestinal and lung parasites in stray dogs and the associated risk factors, as well as providing veterinarians managing shelters useful information for parasite prevention and control.

\section{Materials and Methods}

\section{Study area and sampling}

In the period June 2014-June 2015, 262 stray dogs were captured from an area of Lazio Region (Central Italy) covering 1,814 square kilometres with a population of 476,586 inhabitants (https://www.aslroma5.info/web/) (population density: 262.7 per sq. Km). The study area is characterized by agricultural-rural areas with small towns and villages. The 58.8\% of the land is covered by forest, $32.5 \%$ is used for agricultural activities, $2.5 \%$ is represented by urban areas and $6.2 \%$ is classified as "other use" (Corine Land Cover). After capture, all stray dogs were admitted in a refuge shelter.

\section{Coprological examination}

Each dog was checked for parasites by collecting an individual faecal sample at the moment of its first admission in the shelter, prior to any contact with the other recovered animals. Each faecal sample was examined by a set of analysis, able to detect most of the intestinal and lung parasites: a) the wet mount Lugol's iodine staining, b) the flotation test in a sucrose 1,300 density solution (Taylor et al. 2007), c) the Baerman technique for the detection of lungworms (Bowman 1995; Taylor et al. 2007) and d) a commercial immunofluorescence kit (MERIFLUOR $®$ Cryptosporidium/Giardia, Meridian Bioscience, Inc.) for the detection of Giardia duodenalis and Cryptosporidium spp. For laboratory safety, particularly dealing with the risk due to E. granulosus, endemic in Central Italy (Dakkak 2010), macroscopic examination of faecal samples was not performed; hence, data regarding tapeworm infections must be considered underestimated.

Identification of parasites cysts, oocysts and eggs was morphological (Zajac and Conboy 2012). Lungworms first stage larvae were identified according to McGarry and Morgan (2009). Samples positive for G. duodenalis at microscopy (wet mount Lugol's iodine and/or immunofluorescence) were analysed by molecular tools for genotyping.

\section{G. duodenalis genotyping}

DNA extraction was performed from a group of randomly selected Giardia-positive samples using the QIAamp DNA Stool Mini Kit (Qiagen, Milan, Italy). Samples with less than 130 $\mathrm{mg}$ of feces were excluded from the analysis. A nested endpoint PCR was carried out to amplify a $120 \mathrm{bp}$ fragment of the $18 \mathrm{~S}$ rDNA region, using the specific primers RH11 - RH4 for the first round and the internal primers GIAR-F-GIAR-R for the second round (Read et al. 2002). All PCRs were carried out in a $25 \mu \mathrm{L}$ volume containing $12.5 \mu \mathrm{L}$ PCR master mix 2X (Promega, Milan, Italy), 4-2 $\mu \mathrm{L}$ template DNA (first and second round, respectively), and $0.6 \mathrm{mM}$ of each primer and performed in a TProfessional Basic Thermocycler (Biometra GmbH, Göttingen, Germany). The PCR products were visualized by electrophoresis on $1 \%$ agarose gel stained by SYBR Safe DNA gel stain (Invitrogen, Monza MB, Italy). PCR amplicons were purified using the mi-PCR Purification Kit (Metabion international AG, Planneg, Germany) according to the manufacturer's instructions and directly sequenced by the Bio-Fab Research (Rome, Italy). To assign each isolate to the Assemblage, a comparison of the obtained $18 \mathrm{~S}$ sequences by multiple alignments against sequences retrieved from GenBank database (Accession number: AF199446-Assemblage A; AF199447-Assemblage B; AY775200-Assemblage C; AY775199-Assemblage D; AF113902-Assemblage E; AF199444-Assemblage F; AF199450-Assemblage G) was performed using Clustal Omega.

\section{Risk factors and statistical analysis}

Data regarding the exposure to potential risk factors were collected through a questionnaire filled by Veterinary Authorities at the moment of dog's capture. For each animal, the following data were recorded: date of catch, geographical coordinates and kind of habitat (urban, suburban, agricultural/rural or natural), where the dog was caught, gender, estimated age class ( $<1$ year old, between 1 and 5 years old, $>5$ years old), breed (pedigree or crossbreed), presence/absence of diarrhoea.

Results are presented as absolute frequencies and percentages $(\%)$. Associations between presence/absence of each parasite and the other categorical variables (age, gender, breed, caught area) were evaluated by Chi square test $\left(\chi^{2}\right)$. When more than one risk factor was associated with the presence of a parasite, they were included in a multivariate logistic model to estimate odds ratio (OR) with $95 \%$ confidence interval (CI 95\%). Hosmer and Lemeshow (H-L) test was used to evaluate the model goodness of fit. A p value $<0.05$ was considered statistically significant. All analyses were performed by StataSE v.12.1 for Windows (StataCorp LP, TX, USA). 


\section{Results}

Among the 262 examined dogs, 181 (69.1\%) were positive for at least one parasite. Ninety-four dogs $(51.9 \%)$ were polyparasitized. Two, three, four or five taxa were detected in the $27.6 \%$,

Table I. - Prevalence of parasites detected in stray dogs $(n=262)$ from Central Italy

\begin{tabular}{lcc}
\hline Parasite & $\begin{array}{c}\text { No of } \\
\text { positive dogs }\end{array}$ & Prevalence \% \\
\hline Ancylostomatidae & 106 & 40.5 \\
Giardia duodenalis & 56 & 21.4 \\
Toxocara canis & 54 & 20.6 \\
Trichuris vulpis & 46 & 17.6 \\
Angiostrongylus vasorum & 33 & 12.6 \\
Cystoisospora sp. & 16 & 6.1 \\
Eucoleus aerophilus & 15 & 5.7 \\
Taeniidae & 11 & 4.2 \\
Sarcocystis sp. & 3 & 1.1 \\
Dipylidium caninum & 2 & 0.8 \\
Toxascaris leonina & 2 & 0.8 \\
Crenosoma vulpis & 1 & 0.4 \\
\hline
\end{tabular}

$13.3 \%, 7.7 \%$ and $3.3 \%$ of positive dogs, respectively. Overall, twelve parasitic taxa were detected, three of protozoa and 9 of helminths (Table 1), the helminths being more prevalent than protozoa, $61.8 \%$ and $25.6 \%$ respectively (162 dogs with at least one helminth vs 67 dogs with at least one protozoan).

Concerning protozoa, G. duodenalis was the most prevalent parasite reported (21.4\%). Giardia genotyping obtained by the alignment of the 25 positive samples assigned all isolates to the dog-specific assemblages C (24.0\%) and D (76.0\%).

Concerning intestinal helminths, hookworms of the family Ancylostomatidae were the overall most common recorded parasites $(40.5 \%)$, followed by Toxocara canis $(20.6 \%)$ and Trichuris vulpis (17.6\%). Among lungworms, A. vasorum was the most prevalent $(12.6 \%)$, while Crenosoma vulpis was recorded just in one $\operatorname{dog}(0.4 \%)$. Tapeworms of the family Taeniidae were reported with prevalence of $4.2 \%$ (Table I).

None of the sampled dogs showed diarrhoea at the moment of the introduction in the shelter.

The associations between risk factors and the parasite presence/absence are reported in Table II. No significant association was found between the number of parasite species infecting each dog and the considered variables (age $\chi^{2}=6.56 \mathrm{p}$ $=0.77$, gender $\chi^{2}=9.35 \mathrm{p}=0.11$, breed $\chi^{2}=6.31 \mathrm{p}=0.10$ and capture area $\chi^{2}=22.30 \mathrm{p}=0.10$ ). Taeniidae were associated

Table II. Significant associations between parasites and risk factors

\begin{tabular}{|c|c|c|c|c|c|}
\hline \multirow{2}{*}{ Parasites } & & \multicolumn{2}{|c|}{$\mathrm{N}=262 \mathrm{dogs}$} & \multirow[t]{2}{*}{ Univariate analysis } & \multirow[t]{2}{*}{$\begin{array}{c}\text { Multivariate analysis } \\
\text { OR (CI 95\%) }\end{array}$} \\
\hline & & No of sampled dogs & $\begin{array}{l}\text { No of positive dogs } \\
\text { (Prevalence) }\end{array}$ & & \\
\hline \multicolumn{6}{|l|}{ Taeniidae } \\
\hline \multirow[t]{4}{*}{ Capture area } & Natural & 22 & $4(18.18 \%)$ & \multirow{4}{*}{$\begin{array}{c}\chi^{2}=14.62 \\
p<0.01\end{array}$} & \multirow{4}{*}{-} \\
\hline & Agricultural/Rural & 28 & $0(0 \%)$ & & \\
\hline & Suburban & 126 & $2(1.59 \%)$ & & \\
\hline & Urban & 86 & $5(5.81 \%)$ & & \\
\hline \multicolumn{6}{|l|}{ Trichuris vulpis } \\
\hline \multirow[t]{4}{*}{ Capture area } & Natural & 22 & $8(36.36 \%)$ & \multirow{4}{*}{$\begin{array}{c}\chi^{2}=14.54 \\
p<0.01\end{array}$} & - \\
\hline & Agricultural/Rural & 28 & $10(35.71 \%)$ & & $0.99(0.30-3.32)$ \\
\hline & Suburban & 126 & $17(13.49 \%)$ & & $0.26(0.09-0.74)^{*}$ \\
\hline & Urban & 86 & $11(12.79 \%)$ & & $0.25(0.08-0.77)^{*}$ \\
\hline \multirow[t]{3}{*}{ Age in years ${ }^{\circ}$} & $<1$ & 57 & $3(5.26 \%)$ & \multirow{3}{*}{$\begin{array}{c}\chi^{2}=7.76 \\
p<0.01\end{array}$} & - \\
\hline & $1-5$ & 196 & $41(20.92 \%)$ & & $4.89(1.42-16.77)^{*}$ \\
\hline & $>5$ & 8 & $2(25.00 \%)$ & & $7.06(0.92-53.82)$ \\
\hline \multirow[t]{2}{*}{ Breed } & Pedigree & 227 & $35(15.42 \%)$ & \multirow{2}{*}{$\begin{array}{l}\chi^{2}=5.37 \\
p<0.05\end{array}$} & \multirow{2}{*}{-} \\
\hline & Crossbreed & 35 & $11(31.43 \%)$ & & \\
\hline \multicolumn{6}{|l|}{ Toxocara canis } \\
\hline \multirow[t]{3}{*}{ Age in years ${ }^{\circ}$} & $<1$ & 196 & $26(13.27 \%)$ & \multirow{3}{*}{$\begin{array}{c}\chi^{2}=31.64 \\
\mathrm{p}<0.001\end{array}$} & \multirow{3}{*}{-} \\
\hline & $1-5$ & 57 & $27(47.37 \%)$ & & \\
\hline & $>5$ & 8 & $1(12.5 \%)$ & & \\
\hline \multicolumn{6}{|c|}{ Angiostrongylus vasorum } \\
\hline \multirow[t]{2}{*}{ Gender $^{\circ}$} & Male & 147 & $14(9.52 \%)$ & $\chi^{2}=5.65$ & \multirow{2}{*}{-} \\
\hline & Female & 82 & $17(20.73 \%)$ & $\mathrm{p}<0.05$ & \\
\hline
\end{tabular}

${ }^{\circ}$ Age: 1 missing, Gender: 33 missing. ${ }^{*} \mathrm{p}<0.05$ vs baseline 
with the capture area $(\mathrm{p}<0.01)$, being natural areas where it is most likely to find positive dogs $(18.2 \%)$. T. canis prevalence was significantly lower $(\mathrm{p}<0.001)$ in age class $<1(13.3 \%)$ than $1-5$ years old $(47.4 \%)$. The presence of Ancylostomatidae was significantly associated with $T$. vulpis $(\mathrm{p}<0.05)$ and with A. vasorum ( $\mathrm{p}<0.001)$, being T. vulpis and $A$. vasorum also mutually associated $(\mathrm{p}<0.001)$. A . vasorum was associated with gender $(\mathrm{p}<0.05)$, being females more infected $(20.7 \%)$ than males $(9.5 \%)$. T. vulpis was significantly associated with capture area, age and breed, as detailed in Table II. Considering T. vulpis presence/absence as outcome variable, the first two exposure variables were confirmed as protective/risk factors using a multivariate logistic model $(\mathrm{H}-\mathrm{L}=4.14, \mathrm{p}=0.39)$. In fact, suburban $(\mathrm{OR}=0.26)$ and urban $(\mathrm{OR}=0.25)$ areas resulted significant protective factors $(\mathrm{p}<0.05)$ respect to natural areas, whereas the age class $1-5$ years was a significant risk factor, respect to the $<1$ class $(\mathrm{OR}=4.89, \mathrm{p}<0.05)$. In the multivariate model the influence of breed resulted not significant $(\mathrm{p}=0.126)$ and it was excluded by the final estimates.

\section{Discussion}

Overall, our results agree with those of previous studies conducted in Italy and abroad. Depending on study area, dog category and adopted coprological techniques, roundworms, hookworms, whipworms and G. duodenalis are reported to be the dominant parasites (Capelli et al. 2006; Mohamed et al. 2009; Riggio et al. 2013; Zanzani et al. 2014; Traversa et al. 2017), other ones (Cystoisospora sp., tapeworms, lungworms, Eucoleus spp., etc.), being usually reported with lower prevalence. In our study, we found a higher overall prevalence of helminths than protozoa, similarly to what reported by Riggio et al. (2013).

G. duodenalis prevalence observed in the present study $(21.4 \%)$ is high and confirms the findings of other recent studies both from Italy (Capelli et al. 2006; Paoletti et al. 2015) and from other European countries (Claerebout et al. 2009; Epe et al. 2010; Upjohn et al. 2010). Probably, until recently, the real prevalence of this parasite in dogs was often underestimated (Capelli et al. 2006), because of the low level of awareness of veterinarians through this parasite and the common use of less sensitive diagnostic techniques (Szwabe and Błaszkowska 2017), compared to the immunofluorescence kit adopted in the present study. According to our results, the relevance of stray dogs as possible source of $G$. duodenalis zoonotic infection to humans would be low, as only the specific assemblages $\mathrm{C}$ and $\mathrm{D}$ were identified. Previous surveys on dogs from the same study area reported a $76.5 \%$ (Berrilli et al. 2004) and a $69.3 \%$ (Scaramozzino et al. 2009) of dogspecific genotypes. Other studies in different Italian areas did not report any zoonotic assemblage (Paoletti et al. 2015; Simonato et al, 2015).

Regarding tapeworms of the family Taeniidae, it is impossible to rule out that some of our findings were E. granulosus as eggs in the Taeniidae family are morphologically undistinguishable. The area where the study was carried out is considered endemic for ovine echinococcosis (Dakkak 2010), hence stray dogs are far suspected to be infected.

Nematodes of the family Ancylostomatidae were the most common parasites reported in the present study, with a very high prevalence. Indeed, the study area has environmental conditions particularly favourable to these parasites. The predominant natural-agricultural environment provides microhabitats where free-living larvae are protected from direct sun light and dehydration. Moreover, another possible route of transmission of Ancylostomatidae - the trans-mammary route - could have been favoured by the lack of controls and treatments in the stray dog population.

Not negligible is the prevalence reported for $A$. vasorum $(12.6 \%)$, a parasite considered in geographical expansion in the last ten years (Traversa et al. 2010) and increasing in both wild canids (foxes and wolves) and dogs in the study area (Eleni et al. 2013, 2014). The report of C. vulpis is also remarkable, as this parasite was previously detected only once in our country from the dog (Rinaldi et al. 2007). Foxes are the natural reservoirs of $A$. vasorum and C. vulpis (Barutzki and Schaper 2009), hence their report is not surprising, when considering the mainly rural/natural environments of the sampling area, probably sustaining a high fox population.

In central Italy, two species of the genus Eucoleus are found in dogs, Eucoleus aerophilus and Eucoleus böhmi (De Liberato et al. 2009). The report of E. aerophilus is not surprising, as this species is the most commonly recorded. Regarding observed prevalence, our results are in the range of those reported in a recent paper by Traversa et al. (2017), ranging from $3.0 \%$ to $8.0 \%$.

Despite the overall high parasite prevalence and the report of dogs harboring up to 5 parasitic taxa, no apparent clinical signs were observed in anyone of the tested animals. A high tolerance of dogs to many of the more common parasites is well known from literature (Palmer et al. 2008, Claerebout et al. 2009). However, Riggio et al. (2013) found a positive correlation between the presence of clinical signs and parasitic infection.

The systematic collection of information on dogs and the standardized lab procedures allowed us to explore the association between parasites and dog-environmental variables. Although $T$. canis is usually reported to be more frequent in young dogs respect to older ones (Nijsse et al. 2015), in the present study it resulted significantly more prevalent in dogs $1-5$ years old than $<1$. This apparently surprising result could be related to the different dog categories considered. The owned and well-cared dogs are usually controlled and treated against $T$. canis when few weeks old, while in the present study it is assumable that most part of dogs involved in the present study never received an anthelminthic treatment, hence the prevalence of this nematode would not drop down in adult class of age (Sager et al. 2006). Both Taeniidae and T. vulpis were significantly more frequent in dogs captured in natural areas. This result was expected for Taeniidae, due to their com- 
plex life cycle, but it was quite unexpected for T. vulpis. This nematode was also significantly more common in dogs $1-5$ years old, when compared with younger ones, confirming the majority of comparable studies. A possible explanation can be a cumulative effect during dog lifetime because of the lack of anthelminthic treatments, joined with the high resistance of the eggs in the environment. The observed association between the presence of Ancylostomatidae and T. vulpis is probably ascribable to their common infection route via soil contamination. Despite in published papers no association of $A$. vasorum and host gender was reported, in the present study a significant difference was found between females and males. As it does not seem to be any biological plausibility, we cannot exclude a selection bias due to the passive recruitment of the sampled dogs.

Standing the high parasite prevalence reported at the moment of their introduction in shelters, if not properly managed (control-treatment-post treatment control), stray dogs can constitute a source of infection for dogs already present in the structures and a hazard for zoonosis for people working in shelters. Elsewhere, parasite control in shelters can be very hard to chase, for the high costs for testing and treatments and, in some case the need of repeated treatments (ESCCAP 2010). In general, treatment failures are a common occurrence when dealing with dog parasites, due to re-infection or incorrect application of therapeutic protocols and to the possible appearance of parasites lineages resistant to the most common drugs, as reported for G. duodenalis in human cases (Leitsch 2015).

Parasitized dogs treated at the moment of their introduction in shelters should not be considered parasite-free after a single drug administration and should be tested again before introducing the animal in the shelter in order to minimise environmental contamination (ESCCAP 2010). Parasite control programs are also important to minimize the risk for zoonosis transmission when the animals are adopted by families. The present study provides updated information on the occurrence of parasites in stray dogs from central Italy, confirming that these dogs are a source of environmental contamination with protozoa and helminths of zoonotic concern. Competent authorities should focus efforts on the reduction of the stray dog population and to standardize the sanitary protocols in the dog shelters.

\section{References}

Al-Sabi M.S.N., Kapel C.M.O., Johansson A., Espersen M.C., Koch J., Willesen J.L. 2013. A coprological investigation of gastrointestinal and cardiopulmonary parasites in hunting dogs in Denmark. Veterinary Parasitology, 196, 366-372. DOI: 10.1016/j.vetpar.2013.03.027

ASL ROMA 5, 2017. Accessed at https://www.aslroma5.info/web/ on $17 / 07 / 2017$

Barutzki D., Schaper R. 2009. Natural infection of Angiostrongylus vasorum and Crenosoma vulpis in dogs in Germany. Parasitology Research, 105, 39-48. DOI: 10.1007/s00436-0091494-x
Berrilli F., Di Cave D., De Liberato C., Franco A., Scaramozzino P., Orecchia P. 2004. Genotype characterisation of Giardia duodenalis isolates from domestic and farm animals by SSUrRNA gene sequencing. Veterinary Parasitology, 122, 193-199. DOI: 10.1016/j.vetpar.2004.04.008

Bowman D.D. 1995. Georgi's Parasitology for Veterinarians, 6th ed. (Ed.)W.B. Saunders Company, Philadelphia, 295-296

Capelli G., Frangipane di Regalbono A., Iorio R., Pietrobelli M., Paoletti B., Giangaspero A. 2006. Giardia species and other intestinal parasites in dogs in north-east and central Italy. Veterinary Record, 159, 422-424

Claerebout E., Casaert S., Dalemans A.C., De Wilde N., Levecke B., Vercruysse J., Geurden T. 2009. Giardia and other intestinal parasites in different dog populations in Northern Belgium. Veterinary Parasitology, 161, 41-46. DOI: 10.1016/j.vetpar.2008.11.024

Corine Land Cover. Carta Uso del Suolo della Regione Lazio, Accessed at http://dati.lazio.it/catalog/dataset/cus-lazio on 17 January 2017

Dakkak A. 2010. Echinococcosis/hydatidosis: A severe threat in Mediterranean countries. Veterinary Parasitology, 174, 2-11. DOI: 10.1016/j.vetpar.2010.08.009

De Liberato C., Mazzanti S., Scaramozzino P. 2009. First report of Eucoleus böhmi (Nematoda: Trichuroidea) from Italy: parasitological findings and veterinary implications. Parassitologia, 51, 43-45

Dopchiz M.C., Lavallén C.M., Bongiovanni R., Gonzalez P.V., Elissondo C., Yannarella F., Denegri G. 2013. Endoparasitic infections in dogs from rural areas in the Lobos District, Buenos Aires province, Argentina. Revista Brasileira de Parasitologia Veterinaria, 22, 92-97

Eleni C., De Liberato C., Azam D., Morgan E.R., Traversa D. 2013. Angiostrongylus vasorum in wolves in Italy. International Journal for Parasitology: Parasites and Wildlife, 3, 12-14. DOI: 10.1016/j.ijppaw.2013.10.003

Eleni C., Grifoni G., Di Egidio A., Meoli R., De Liberato C. 2014. Pathological findings of Angiostrongylus vasorum infection in red foxes (Vulpes vulpes) from central Italy, with the first report of a disseminated infection in this host species. Parasitology Research, 113, 1247-1250. DOI: 10.1007/s00436014-3793-0

Epe C., Rehkter G., Schnieder T., Lorentzen L., Kreienbrock L. 2010. Giardia in symptomatic dogs and cats in Europe: results of a European study. Veterinary Parasitology, 173, 32-38. DOI: 10.1016/j.vetpar.2010.06.015

ESCCAP (European Scientific Counsel Companion Animal Parasites). 2010. Guideline No. 1. Worm Control in Dogs and Cats Second Edition. Accessed at http://www.esccap.org/uploads/docs/nkzqxmxn_esccapgl1endoguidelines.pdf on 17 January 2017

Leitsch D. 2015. Drug resistance in the microaerophilic parasite $G i$ ardia lamblia. Current Tropical Medicine Reports, 2, 128135. DOI: $10.1007 / \mathrm{s} 40475-015-0051-1$

Little S.E., Johnson E.M., Lewis D., Jaklitsch R.P., Payton M.E., Blagburn B.L., et al. 2009. Prevalence of intestinal parasites in pet dogs in the United States. Veterinary Parasitology, 166, 144-152. DOI: 10.1016/j.vetpar.2009.07.044.

Martins C.M., da Conceiçáo de Barros C., Bier D., Marinho A.P., Gonçalves Figueiredo J.M., Hoffmann J.L., Molento M.B., Biondo A.W. 2012. Dog parasite incidence and risk factors, from sampling after one-year interval, in Pinhais, Brazil. Revista Brasileira de Parasitologia Veterinaria, 21, 101106

Mateus T.L., Castro A., Ribeiro J.N., Vieira-Pinto M. 2014. Multiple zoonotic parasites identified in dog feces collected in Ponte de Lima, Portugal - A potential threat to human health. Interna- 
tional Journal of Environmental Research and Public Health, 11, 9050-9067. DOI: 10.3390/ijerph110909050

McGarry J.W., Morgan E.R. 2009. Identification of first-stage larvae of metastrongyles from dogs. Veterinary Record, 165, 258261

Mirzaei M., Fooladi M. 2012. Prevalence of intestinal helminthes in owned dogs in Kerman city, Iran. Asian Pacific Journal of Tropical Medicine, 20, 735-737. DOI: 10.1016/S19957645(12)60116-3

Mohamed A.S., Moore G.E., Glickman L.T. 2009. Prevalence of intestinal nematode parasitism among pet dogs in the United States (2003-2006). Journal of the American Veterinary Medicine Association, 234, 631-637. DOI: 10.2460/javma.234.5.631

Nijsse R., Ploeger H.W., Wagenaar J.A., Mughini-Gras L. 2015. Toxocara canis in household dogs: prevalence, risk factors and owner's attitude towards deworming. Parasitology Research, 114, 561-569. DOI: 10.1007/s00436-014-4218-9

Palmer C.S., Thompson R.C.A., Traub R.J., Rees R., Robertson I.D. 2008. National studies of the gastrointestinal parasites of dogs and cats in Australia. Veterinary Parasitology, 151, 181-190. DOI: 10.1016/j.vetpar.2007.10.015

Paoletti B., Traversa D., Iorio R., De Berardinis A., Bartolini R., Salini R., Di Cesare A. 2015. Zoonotic parasites in feces and fur of stray and private dogs from Italy. Parasitology Research, 114, 2135-2141. DOI: 10.1007/s00436-015-4402-6

Read C., Walters J., Robertson I.D., Thompson R.C.A. 2002. Correlation between genotype of Giardia duodenalis and diarrhoea. International Journal for Parasitology, 32, 229-231

Riggio F., Mannella R., Ariti G., Perrucci S. 2013. Intestinal and lung parasites in owned dogs and cats from central Italy. Veterinary Parasitology, 193, 78-84. DOI: 10.1016/j.vetpar.2012.11.026

Rinaldi L., Calabria G., Carbone S., Carrella A., Cringoli G. 2007. Crenosoma vulpis in dog: first case report in Italy and use of the FLOTAC technique for copromicroscopic diagnosis. $\mathrm{Pa}$ rasitology Research, 101, 1681-1684. DOI: 10.1007/s00436007-0713-6

Sager H., Steiner Moret C., Grimm F., Deplazes P., Doherr M.G., Gottstein B. 2006. Coprological study of intestinal helminths in Swiss dogs: temporal aspects of anthelminthic treatment. Parasitology Research, 98, 333-338. DOI: 10.1007/s00436005-0093-8

Received: July 12, 2017

Revised: August 28, 2017

Accepted for publication: September 14, 2017
Scaramozzino P., Di Cave D., Berrilli B., D’Orazi C., Spaziani A., Mazzanti S., et al. 2009. A study of the prevalence and genotypes of Giardia duodenalis infecting kennelled dogs. Veterinary Journal, 182, 231-234. DOI: 10.1016/j.tvj1.2008.07.003

Simonato G., Frangipane di Regalbono A., Cassini R., Traversa D., Beraldo P., et al. 2015. Copromicroscopic and molecular investigations on intestinal parasites in kennelled dogs. Parasitology Research, 114, 1963-1970. DOI: 10.1007/s00436015-4385-3

Szwabe K., Błaszkowska J. 2017. Stray dogs and cats as potential sources of soil contamination with zoonotic parasites. Annals of Agriculture and Environmental Medicine, 24, 39-43. DOI: $10.5604 / 12321966.1234003$

Taylor M.A., Coop R.L., Wall R.L. 2007. Veterinary Parasitology, 3rd edition. (Ed.) Blackwell Publishing, Oxford, UK

Traversa D., Di Cesare A., Conboy G. 2010. Canine and feline cardiopulmonary parasitic nematodes in Europe: emerging and underestimated. Parasites and Vectors, 3, 62-84. DOI: 10.1186/1756-3305-3-62

Traversa D., Di Cesare A., Simonato G., Cassini R., Merola C., Diakou A., et al. 2017. Zoonotic intestinal parasites and vector-borne pathogens in Italian shelter and kennel dogs. Comparative Immunology, Microbiology and Infectious Disease, 51, 69-75. DOI: 10.1016/j.cimid.2017.04.003

Upjohn M., Cobb C., Monger J., Geurden T., Claerebout E., Fox M. 2010. Prevalence, molecular typing and risk factor analysis for Giardia duodenalis infections in dogs in a central London rescue shelter. Veterinary Parasitology, 172, 341-346. DOI: 10.1016/j.vetpar.2010.05.010

Zajac A.M., Conboy G. 2012. Veterinary clinical parasitology. 8th edition. (Ed.) Wiley-Blackwell Publishing, Oxford, UK

Zanzani S.A., Gazzonis A.L., Scarpa P., Berrilli F., Manfredi M.T. 2014. Intestinal parasites of owned dogs and cats from metropolitan and micropolitan areas: prevalence, zoonotic risks and pet owner awareness in Northern Italy. BioMed Reseach International, 2014, 1-10. DOI: 10.1155/2014/696508 